\title{
Scheduling jobs with hard deadlines over Multiple Access and Degraded Broadcast Channels
}

\author{
Dinkar Vasudevan, Vijay G. Subramanian and Douglas J. Leith \\ Hamilton Institute, National University of Ireland, Maynooth
}

\begin{abstract}
We consider the problem of scheduling jobs with given start and finish times over two classes of multi-user channels, namely Multiple Access Channels and Degraded Broadcast Channels, and derive necessary and sufficient conditions for feasible scheduling of the jobs.
\end{abstract}

\section{INTRODUCTION}

We consider the problem of scheduling jobs with hard deadlines over Multiple Access Channels (MAC) and Degraded Broadcast Channels (DBC). The jobs correspond to independent information bits and the hard deadlines correspond to given start and finish times within which the bits must be communicated to the receiver. The problem was formulated by Martel [1] who studied the premptive scheduling of jobs with hard deadlines among machines with differing service times. He showed that the scheduling problem is equivalent to determining the maximum flow over a suitably defined polymatroidal flow network, and proposed an algorithm to find the maximum flow. In this paper, we define and solve Martel's scheduling problem for multi-user channels.

\section{Problem Statement And Related Work}

We first define the problem for the $M$ user MAC. For ease of exposition we assume that there are $M$ jobs, one at each transmitter. The job at transmitter $i$ comprises of $w_{i}$ bits that have to be communicated reliably to the receiver. The job has a start time $s_{i}$, corresponding to when the job becomes available for transmission, and a deadline $d_{i}$, corresponding to the time by which the bits must be communicated reliably to the receiver. We assume there is a centralized scheduler to which the transmitters communicate the job details, i.e., $\left(w_{i}, s_{i}, d_{i}\right)$ ahead of time. The scheduling problem is to determine if it is feasible to schedule the jobs, and if so, to construct the scheduling. The problem can similarly be defined for the $M$ user DBC. In this case, all the $M$ jobs are colocated at the transmitter, and each job has to be communicated to a distinct receiver within the deadline. For simplicity, in the DBC case we only consider $M=2$ with both the jobs having the same start time; this corresponds to the case when the transmitter has both the jobs at the start.

For both problems, we derive the necessary and sufficient conditions for feasibility of scheduling. The conditions are described by bounds on the rates of the job, that are functions of the underlying channel law. The solution for the scheduling problem for the MAC is obtained by using the idea of Martel, namely that of constructing an equivalent polymatroidal flow network and determining the maximum flow over this network.
The solution for scheduling over the DBC follows using superposition coding [2] arguments.

Scheduling problems have been studied extensively for single or multiuser channels from the point of view of energy efficiency (see [3][4] [5] [6] [7]). Typically in these works, the problem is that of determining a schedule which minimizes the total energy consumed over the transmission while respecting the deadline constraints. The channels considered are multiuser Gaussian channels or channels where the power is convex in the transmission rates. In contrast, our study applies to the larger class of discrete memoryless multi-user channels, where we fix the channel input distribution (equivalently the average power in the Gaussian case) over the entire period of transmission and determine if scheduling is feasible.

The organization of the paper is as follows: Section III sets up the notation. In Section IV and V, respectively, we prove the necessary and sufficient conditions for feasible scheduling over the MAC. The Appendix contains some background results on polymatroidal flow networks which will be useful in the proof of the sufficiency. In Section VI, we prove the feasibility conditions for the 2 receiver DBC.

\section{NOTATION AND PRELIMINARIES}

Let $X_{j}$ denote the input of the $j^{\text {th }}$ transmitter and $Y$ denote the output at the receiver of the MAC. The channel law is given by $p_{Y \mid X_{1}, \ldots X_{M}}(\cdot \mid \cdot)$. Time is slotted and we use the start and finish times of the jobs to divide the timeline into $2 M-1$ intervals. Let $t_{i}$ be the $i^{\text {th }}$ smallest value among the $2 M$ start and finish times $\left\{s_{1}, \ldots, s_{M}, d_{1}, \ldots, d_{M}\right\}$. The $i^{\text {th }}$ interval or epoch is the time period from $t_{i}$ to $t_{i+1}$. Let $\Delta_{i}=t_{i+1}-t_{i}$. The $j^{\text {th }}$ job is said to be active in the $i^{\text {th }}$ interval iff $s_{j} \leq t_{i}$ and $d_{j} \geq t_{i+1}$. Note that within an interval, the set of active jobs does not change. We let $\mathcal{N}(i)$ denote the set of transmitters whose jobs are active during the interval $i$ and let $\mathcal{D}(i)=$ $\mathcal{N}(i)^{c}$ denote the set of transmitters whose jobs are not active in the interval $i$. Likewise for every job $j$, we denote by $\mathcal{N}(j)$ the time intervals during which the job $j$ is active.

We assume that each $\Delta_{i}$ is an integer and define $\Delta=$ $\sum_{i} \Delta_{i}$. Let the vectors $\mathbf{X}_{j}$ and $\mathbf{Y}$ denote the (size $\Delta$ ) block input of transmitter $j$ and output respectively of the MAC. Let $\mathbf{X}_{j, i}$ and $\mathbf{Y}_{i}$ denote the (size $\Delta_{i}$ ) block input of transmitter $j$ and the MAC output in the $i^{\text {th }}$ interval. We say that $\mathbf{X}_{j, i}$ is a dummy input in the interval $i$ if $j \in \mathcal{D}(i)$. The dummy inputs of every transmitter are assumed to be known to the receiver but not to the other transmitters of the MAC. We re-emphasize that all transmitters have signaled their job details $\left\{\left(w_{i}, s_{i}, d_{i}\right)\right\}_{1}^{M}$ 
to the receiver at the start of time. The receiver thus knows $\mathcal{N}(i), \mathcal{D}(i)$ for every interval $i$. The dummy inputs do not carry any information and act as sideinformation at the receiver. We denote the vector of dummy inputs to interval $i$ by $\mathbf{X}_{\mathcal{D}(i), i}$ (consisting of all the block inputs $\mathbf{X}_{j, i}$ that are dummy inputs) and denote the vector of dummy inputs across all intervals by $\mathbf{X}_{\mathcal{D}}$. We denote by $\mathbf{X}_{\mathcal{J}}$, the inputs corresponding to the transmitters $j \in \mathcal{J}$ in the interval $i$. The set of intervals in which any job in $\mathcal{J}$ is active is denoted by $\mathcal{N}(\mathcal{J})$ and we define

$$
\mathbf{Y}_{\mathcal{N}(\mathcal{J})} \triangleq\left\{\mathbf{Y}_{i}: i \in \mathcal{N}(\mathcal{J})\right\}
$$

Thus $\mathbf{Y}_{\mathcal{N}(\mathcal{J})}$ denotes the block outputs in intervals where jobs in $\mathcal{J}$ are active.

\section{NECESSARY CONDITION FOR SCHEDULING}

We derive a necessary condition for feasible scheduling given the job size $w_{j}$ 's, based on information-theoretic converse arguments.

Theorem 1. A necessary condition for the feasible scheduling of jobs is if there exist random variables $\left(U_{i}, X_{j, i}\right)$ for all $i=1, \ldots, 2 M-1$ and $j=1 \ldots, M$ distributed as $\prod_{i} p_{U_{i}, X_{\mathcal{D}(i), i}}(\cdot, \cdot) \prod_{j \in \mathcal{N}(i)} p_{X_{j, i} \mid U_{i}}(\cdot \mid \cdot)$ such that for every $\mathcal{J} \subseteq\{1, \ldots, M\}$

$$
\frac{\sum_{j \in \mathcal{J}} w_{j}}{\Delta}<\sum_{i \in \mathcal{N}(\mathcal{J})} \frac{\Delta_{i}}{\Delta} I\left(Y_{i} ; X_{\mathcal{J}, i} \mid X_{\mathcal{J}^{c}, i}, X_{\mathcal{D}(i), i}, U_{i}\right)
$$

By Caratheodeory's theorem [2], it suffices to choose $\left|\mathcal{U}_{i}\right|=$ $2^{|\mathcal{N}(i)|}$.

Proof: Let $W_{j}$ represent the message of transmitter $j$ having entropy $w_{j}$. Let $P_{e}$ denote the probability of error in decoding any of messages. From Fano's inequality, it follows that for all $\mathcal{J} \subseteq\{1, \ldots, M\}$,

$$
H\left(W_{\mathcal{J}} \mid \mathbf{Y}_{\mathcal{N}(\mathcal{J})}, W_{\mathcal{J}^{c}}, \mathbf{X}_{\mathcal{D}}\right) \leq P_{e} \sum_{j=1}^{M} w_{j}+H\left(P_{e}\right) \triangleq \Delta \epsilon
$$

In the above, $\epsilon \rightarrow 0$ as $P_{e} \rightarrow 0$. Consider any subset $\mathcal{J} \subseteq$ $\{1, \ldots, M\}$ of jobs. The entropy of messages in $\mathcal{J}$ can be bounded as

$$
\begin{aligned}
& \sum_{j \in \mathcal{J}} w_{j}=H\left(W_{\mathcal{J}}\right) \stackrel{(a)}{=} H\left(W_{\mathcal{J}} \mid W_{\mathcal{J}^{c}}, \mathbf{X}_{\mathcal{D}}\right) \\
& =I\left(W_{\mathcal{J}} ; \mathbf{Y}_{\mathcal{N}(\mathcal{J})} \mid W_{\mathcal{J}^{c}}, \mathbf{X}_{\mathcal{D}}\right)+H\left(W_{\mathcal{J}} \mid \mathbf{Y}_{\mathcal{N}(\mathcal{J})}, W_{\mathcal{J}^{c}}, \mathbf{X}_{\mathcal{D}}\right) \\
& \leq I\left(W_{\mathcal{J}} ; \mathbf{Y}_{\mathcal{N}(\mathcal{J})} \mid W_{\mathcal{J}^{c}}, \mathbf{X}_{\mathcal{D}}\right)+\Delta \epsilon \\
& =H\left(\mathbf{Y}_{\mathcal{N}(\mathcal{J})} \mid W_{\mathcal{J}^{c}}, \mathbf{X}_{\mathcal{D}}\right)-H\left(\mathbf{Y}_{\mathcal{N}(\mathcal{J})} \mid W_{\mathcal{J}^{c}}, W_{\mathcal{J}}, \mathbf{X}_{\mathcal{D}}\right) \\
& +\Delta \epsilon \\
& \stackrel{(b)}{\leq} \sum_{i \in \mathcal{N}(\mathcal{J})} H\left(\mathbf{Y}_{i} \mid \mathbf{X}_{\mathcal{J}^{c}, i}, \mathbf{X}_{\mathcal{D}(i), i}\right) \\
& -H\left(\mathbf{Y}_{\mathcal{N}(\mathcal{J})} \mid W_{\mathcal{J}^{c}}, W_{\mathcal{J}}, \mathbf{X}_{\mathcal{D}}\right)+\Delta \epsilon \\
& \stackrel{(c)}{=} \sum_{i \in \mathcal{N}(\mathcal{J})} H\left(\mathbf{Y}_{i} \mid \mathbf{X}_{\mathcal{J}^{c}, i}, \mathbf{X}_{\mathcal{D}(i), i}\right)
\end{aligned}
$$

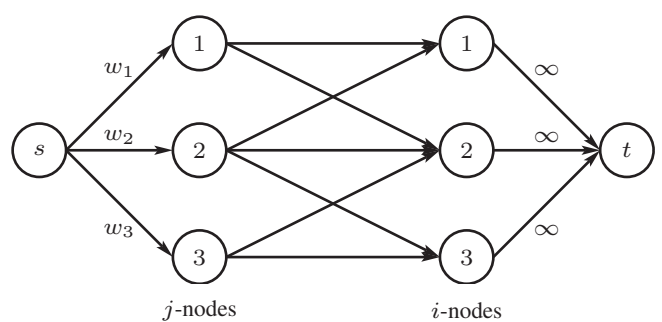

Fig. 1. Graph with $3 j$-nodes and $3 i$-nodes. $\left|\mathcal{U}_{i}\right|=1$ for $i=1,2,3$.

$$
\begin{aligned}
& -\sum_{i \in \mathcal{N}(\mathcal{J})} H\left(\mathbf{Y}_{i} \mid \mathbf{X}_{1, i}, \ldots, \mathbf{X}_{M, i}\right)+\Delta \epsilon \\
& =\sum_{i \in \mathcal{N}(\mathcal{J})} I\left(\mathbf{Y}_{i} ; \mathbf{X}_{\mathcal{J}, i} \mid \mathbf{X}_{\mathcal{J}^{c}, i}, \mathbf{X}_{\mathcal{D}(i), i}\right)+\Delta \epsilon \\
& \stackrel{(d)}{\leq} \sum_{i \in \mathcal{N}(\mathcal{J})} \sum_{l=1}^{\Delta_{i}} I\left(Y_{i}(l) ; X_{\mathcal{J}, i}(l) \mid X_{\mathcal{J}^{c}, i}(l), X_{\mathcal{D}(i), i}(l)\right) \\
& +\Delta \epsilon \\
& =\sum_{i \in \mathcal{N}(\mathcal{J})} \Delta_{i} I\left(Y_{i}\left(U_{i}\right) ; X_{\mathcal{J}, i}\left(U_{i}\right) \mid X_{\mathcal{J}^{c}, i}\left(U_{i}\right), X_{\mathcal{D}(i), i}\left(U_{i}\right), U_{i}\right)
\end{aligned}
$$$$
+\Delta \epsilon
$$

where in the last expression, the symbols $Y_{i}(l), X_{\mathcal{J}, i}(l), X_{\mathcal{J}^{c}, i}(l), X_{\mathcal{D}(i), i}(l)$ denote the $l^{\text {th }}$ symbol in the corresponding block. We explain the inequalities above: (a) follows from the independence of the messages and the dummy codewords, (b) follows since removing the "conditioned on" terms cannot decrease entropy, (c) follows from the memoryless property of the MAC and (d) follows from the same reasoning as (b) and (c). The last equality follows from defining the random variable $U_{i}$ for all $i$, which takes values uniformly in the integer set $\left\{1, \ldots, \Delta_{i}\right\}$. It can be verified that the distribution $p_{X_{\mathcal{N}(i), i}\left(U_{i}\right), X_{\mathcal{D}(i), i}\left(U_{i}\right), U_{i}}$ decouples as $p_{U_{i}} p_{X_{\mathcal{D}(i), i} \mid U_{i}} \prod_{j \in \mathcal{N}(i)} p_{X_{j, i} \mid U_{i}}$.

\section{Proof of SUFFICIENCY}

In this section, we prove the sufficiency of the necessary condition (1) for feasible scheduling. The proof is via polymatroidal network flows.

Theorem 2. The necessary condition (1) for feasible scheduling is also sufficient.

Proof: Fix the input distribution: $\prod_{i} p_{U_{i}, X_{\mathcal{D}(i), i}}(\cdot, \cdot) \prod_{j \in \mathcal{N}(i)} p_{X_{j, i} \mid U_{i}}(\cdot \mid \cdot)$. We define a directed graph $\mathcal{G}=(\mathcal{V}, \mathcal{E})$ with set $\mathcal{V}$ of vertices and set $\mathcal{E}$ of edges . There is a $j$-node for each job and $\left|\mathcal{U}_{i}\right|$ nodes (labeled $i_{1}, \ldots i_{\left|\mathcal{U}_{i}\right|}$ ) for the $i^{\text {th }}$ interval for $i=1, \ldots, 2 M-1$. There is an edge directed from node $j$ to node $i_{u}$ if the job $j$ is active in the interval $i$. There is also a source node $s$ with an arc from $s$ to each $j$-node of capacity $w_{j}$ and a sink node $t$ with an arc from each $i_{u}$-node to $t$ of capacity $\infty$. An example of such a network is illustrated in Figure 1. With respect to any node $l$, we denote the set of incoming edges by $I_{l}$ and the set of outgoing edges by $O_{l}$. 
A flow in the network is an assignment of real numbers to the edges of the network. We let the flow be represented by a function $f: \mathcal{E} \rightarrow \mathbb{R}^{+}$which extends to sets of $\mathcal{E}$ in a natural way, i.e.,

$$
\begin{aligned}
f(\phi) & =0, \\
f(A) & =\sum_{e \in A} f(e) \quad(\phi \neq A \subseteq \mathcal{E})
\end{aligned}
$$

The flow has the following interpretation with respect to the scheduling problem: The flow $f(j, i)$ from a $j$-node to an $i$ node implies that $f(j, i)$ bits of the job $j$ are communicated reliably to the receiver in the interval $i$. The flow function is said to be feasible if

$$
\begin{aligned}
f(s, j) & \leq w_{j} \quad \forall j=1, \ldots, M \\
f(A) & \leq I\left(Y_{i} ; X_{\mathcal{J}, i} \mid X_{\mathcal{J}^{c}, i}, X_{\mathcal{D}(i), i}, U_{i}=u_{i}\right) p\left(u_{i}\right) \Delta_{i} \\
\forall i, A \subseteq I_{i_{u_{i}}}, \mathcal{J} \triangleq\{j:(j, i) \in A\} & \\
f(s, j) & =f\left(O_{j}\right) \quad j=1, \ldots, M \\
f\left(I_{i_{u}}\right) & =f\left(i_{u}, t\right) \quad i=1, \ldots, 2 M-1, u=1, \ldots,\left|\mathcal{U}_{i}\right| \\
f(e) & \geq 0 \quad \forall e \in \mathcal{E}
\end{aligned}
$$

The mutual information term in (3) is computed with respect to the input distribution $p_{X_{\mathcal{D}(i), i} \mid U_{i}}\left(\cdot \mid u_{i}\right) \prod_{j \in \mathcal{N}(i)} p_{X_{j, i} \mid U_{i}}\left(\cdot \mid u_{i}\right)$ defined for every interval $i$ and every $u_{i}$. The inequality (2) ensures that at most $w_{j}$ bits are assigned to the job $j$. The inequality (3) ensures that the rates assigned to the transmitters in interval $i$ for each subinterval $i_{u_{i}}$ corresponding to the choice of input distribution $\prod_{i} p_{U_{i}, X_{\mathcal{D}(i), i}}\left(u_{i}, \cdot\right) \prod_{j \in \mathcal{N}(i)} p_{X_{j, i} \mid U_{i}}\left(\cdot \mid u_{i}\right)$ are achievable (We assume that the $\Delta_{i}$ 's are large enough so that in each subinterval of duration $p\left(u_{i}\right) \Delta_{i}$, the bits can be transmitted at any rate inside the MAC capacity region corresponding to the input distribution for the subinterval). The equalities (4) and (5) ensure flow conservation respectively at the $j$-nodes and $i$ nodes. Finally, (6) requires that the flow through each edge be non-negative.

It follows that any feasible flow corresponds to a schedule. In particular, note that the value of the net flow between the source and the sink is the amount of processing completed in the schedule corresponding to the flow. Thus, a feasible schedule which completes all jobs exists if there exists a flow in the network defined by (2) - (6) with net value $\sum_{j=1}^{m} w_{j}$. The sufficiency condition therefore, is one which ensures that the max flow in the above defined flow network has value $\sum_{j=1}^{m} w_{j}$.

It can be checked that the flow network defined as in (2) - (6) falls in the class of "polymatroidal" flow networks, i.e., networks with submodular capacity constraints on sets of edges (see Appendix for definitions and results on the max-flow min-cut theorem for polymatroidal flow networks). Consider a partition of the set of vertices $\mathcal{V}$ into two sets $\left(\mathcal{W}, \mathcal{W}^{c}\right)$ such that $s \in \mathcal{W}$ and $t \in \mathcal{W}^{c}$ and let $\tilde{\mathcal{E}} \subset \mathcal{E}$ be the edges which are directed from $\mathcal{W}$ to $\mathcal{W}^{c}$. The cut $C\left(\mathcal{W}, \mathcal{W}^{c}\right)$ is evaluated as (see Appendix)

$$
C\left(\mathcal{W}, \mathcal{W}^{c}\right)=\left\{\begin{array}{l}
\infty \quad \text { if } \exists i_{u} \text { s.t. }\left(i_{u}, t\right) \in \tilde{\mathcal{E}} \\
\sum_{j=1}^{M} w_{j} 1_{(s, j) \in \tilde{\mathcal{E}}} \\
+\sum_{i=1}^{2 M-1} I\left(Y_{i} ; X_{\mathcal{W}, i} \mid X_{\mathcal{W}^{c}, i}, X_{\mathcal{D}(i), i}, U_{i}\right) \Delta_{i} \\
\text { else }
\end{array}\right.
$$

From the max-flow min-cut theorem for polymatroidal flow networks [8], it holds that the maximum feasible flow from $s$ to $t$ is equal to the $\min -\operatorname{cut}(s, t) \triangleq \min _{\mathcal{W} \in \mathcal{V}} C\left(\mathcal{W}, \mathcal{W}^{c}\right)$. Thus, a sufficient condition is one which ensures that min-cut $(s, t)=$ $\sum_{j=1}^{m} w_{j}$. Note that the cut separating just $s$ from all the remaining vertices has value $\sum_{j=1}^{m} w_{j}$. Therefore, it only remains to show that the value of any other cut is at least $\sum_{j=1}^{M} w_{j}$. This is clearly the case if the cut includes an edge $\left(i_{u}, t\right)$. Otherwise, it must be that for every subset $\mathcal{J} \subset\{1, \ldots, M\}$, the cut separating the vertices in $\mathcal{J}$ from the vertices $\mathcal{J}^{c}$ is at least $\sum_{j=1}^{M} w_{j}$. Thus, we have

$$
\begin{aligned}
\sum_{j \in \mathcal{J}^{c}} w_{j}+\sum_{j \in \mathcal{J}} w_{j} & \leq \sum_{j \in \mathcal{J}^{c}} w_{j} \\
& +\sum_{i=1}^{2 M-1} I\left(Y_{i} ; X_{\mathcal{J}, i} \mid X_{\mathcal{J}^{c}, i}, X_{\mathcal{D}(i), i}, U_{i}\right) \Delta_{i}
\end{aligned}
$$

which is just the necessary condition (1).

Remark 3. We emphasize that apart from proving the sufficiency of the necessary condition, the Lawler-Martel algorithm [8] finds the operating rates of the transmitters in every interval in the feasible schedule.

Remark 4. If there is a only one interval where all jobs are active, we recover the capacity region of the MAC.

\section{SCHEDUling OVER A 2 RECEIVER DEGRADED BROADCAST CHANNEL(DBC)}

Consider a 2-receiver DBC. There are two jobs at the transmitter, one for each receiver. We assume both jobs have the same start time, but possibly different deadlines. The setup is illustrated in Figure 2. The two nodes on the left represent the two receivers while the two nodes on the right represent the two intervals $\Delta_{i}$ for $i=1,2$. An arrow from a receiver to an interval indicates that the corresponding job at the receiver is active in the interval. In Figure $2, \Delta_{1}$ corresponds to the time interval when both jobs are active and $\Delta_{2}$ corresponds to the interval when only the job corresponding to receiver 2 is active. Let the input of the transmitter in interval $i$ be $\mathbf{X}_{i}$ and the corresponding outputs at receivers 1 and 2 by $\mathbf{Y}_{i}$ and $\mathbf{Z}_{i}$. The receiver 1 decodes message $W_{1}$ based on observations $\mathbf{Y}_{1}$ while receiver 2 decodes message $W_{2}$ based on observations $\left(\mathbf{Z}_{1}, \mathbf{Z}_{2}\right)$. In each interval $i$, the input $\mathbf{X}_{i}$ is (in general) a function of both messages.

We consider the two cases when $X \odot Y \ominus Z$ and $X \ominus Z \ominus Y$, the degradations being stochastic in general. We first derive the necessary and sufficient conditions for the case $X \ominus Z \bullet Y$. This is the case where the stronger receiver has a later decoding deadline as compared to the weaker receiver. 


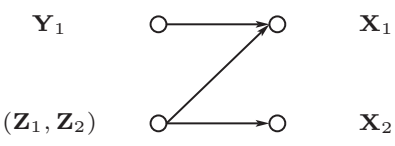

Fig. 2.

Theorem 5. A necessary and sufficient condition for feasible scheduling is if $\left(\frac{w_{1}}{\Delta}, \frac{w_{2}}{\Delta}\right)$ lies in the closure of regions defined by

$$
\begin{aligned}
& \frac{w_{1}}{\Delta}<\frac{\Delta_{1}}{\Delta} I^{(1)}(U ; Y) \\
& \frac{w_{2}}{\Delta}<\frac{\Delta_{1}}{\Delta} I^{(1)}(X ; Z \mid U)+\frac{\Delta_{2}}{\Delta} I^{(2)}(X ; Z)
\end{aligned}
$$

where the mutual informations $I^{(1)}, I^{(2)}$ are evaluated with respect to the distributions $p_{U, X}^{(1)}(\cdot, \cdot)$ and $p_{X}^{(2)}(\cdot)$ respectively. By Caratheodeory's theorem ([2]), it suffices to choose $\left|\mathcal{U}_{i}\right|=$ $\min (|\mathcal{X}|,|\mathcal{Y}|,|\mathcal{Z}|)$.

Proof: The proof of necessity follows using similar steps as in the proof of the converse of the DBC capacity region [2]. The message entropy $W_{1}$ can be bounded as

$$
\begin{aligned}
& H\left(W_{1}\right)=I\left(W_{1} ; \mathbf{Y}_{1}\right)+H\left(W_{1} \mid \mathbf{Y}_{1}\right) \\
& \quad \leq I\left(W_{1} ; \mathbf{Y}_{1}\right)+\Delta_{1} \epsilon_{1}=\sum_{i=1}^{\Delta_{1}} I\left(W_{1} ; \mathbf{Y}_{1 i} \mid \mathbf{Y}_{1}^{i-1}\right)+\Delta_{1} \epsilon_{1} \\
& \quad \leq \sum_{i=1}^{\Delta_{1}} I\left(W_{1}, \mathbf{Y}_{1}^{i-1} ; \mathbf{Y}_{1 i}\right)+\Delta_{1} \epsilon_{1} \\
& \quad \stackrel{(a)}{\leq} \sum_{i=1}^{\Delta_{1}} I\left(W_{1}, \mathbf{Z}_{1}^{i-1} ; \mathbf{Y}_{1 i}\right)+\Delta_{1} \epsilon_{1} \\
& \quad \stackrel{(b)}{=} \sum_{i=1}^{\Delta_{1}} I\left(U_{i} ; \mathbf{Y}_{1 i}\right)+\Delta_{1} \epsilon_{1} .
\end{aligned}
$$

The inequality (a) follows since $\left(W_{1}, \mathbf{Y}_{1}^{i-1}\right) \quad \ominus$ $\left(W_{1}, \mathbf{Z}_{1}^{i-1}\right) \quad \ominus \quad \mathbf{Y}_{1 i}$ and (b) follows by defining $U_{i} \triangleq\left(W_{1}, \mathbf{Z}_{1}^{i-1}\right)$. The message entropy $W_{2}$ can be bounded as

$$
\begin{aligned}
& H\left(W_{2}\right)=H\left(W_{2} \mid W_{1}\right) \\
& =I\left(W_{2} ; \mathbf{Z}_{1}, \mathbf{Z}_{2} \mid W_{1}\right)+H\left(W_{2} \mid \mathbf{Z}_{1}, \mathbf{Z}_{2}, W_{1}\right) \\
& \leq I\left(W_{2} ; \mathbf{Z}_{1}, \mathbf{Z}_{2} \mid W_{1}\right)+\Delta \epsilon_{2} \\
& =I\left(W_{2} ; \mathbf{Z}_{1} \mid W_{1}\right)+I\left(W_{2} ; \mathbf{Z}_{2} \mid \mathbf{Z}_{1}, W_{1}\right)+\Delta \epsilon_{2} \\
& =\sum_{i=1}^{\Delta_{1}} I\left(W_{2} ; \mathbf{Z}_{1 i} \mid U_{i}\right)+\sum_{i=1}^{\Delta_{2}}\left(H\left(\mathbf{Z}_{2 i} \mid \mathbf{Z}_{2}^{i-1}, \mathbf{Z}_{1}, W_{1}\right)\right. \\
& \left.-H\left(\mathbf{Z}_{2 i} \mid \mathbf{Z}_{2}^{i-1}, \mathbf{Z}_{1}, W_{1}, W_{2}\right)\right)+\Delta \epsilon_{2} \\
& \leq \sum_{i=1}^{\Delta_{1}} I\left(W_{2} ; \mathbf{Z}_{1 i} \mid U_{i}\right)+\sum_{i=1}^{\Delta_{2}}\left(H\left(\mathbf{Z}_{2 i}\right)\right. \\
& \left.-H\left(\mathbf{Z}_{2 i} \mid \mathbf{Z}_{2}^{i-1}, \mathbf{Z}_{1}, W_{1}, W_{2}\right)\right)+\Delta \epsilon_{2}
\end{aligned}
$$

$$
\begin{aligned}
& =\sum_{i=1}^{\Delta_{1}} I\left(W_{2} ; \mathbf{Z}_{1 i} \mid U_{i}\right)+\sum_{i=1}^{\Delta_{2}}\left(H\left(\mathbf{Z}_{2 i}\right)\right. \\
- & \left.H\left(\mathbf{Z}_{2 i} \mid \mathbf{Z}_{2}^{i-1}, \mathbf{Z}_{1}, W_{1}, W_{2}, \mathbf{X}_{2 i}\right)\right)+\Delta \epsilon_{2} \\
= & \sum_{i=1}^{\Delta_{1}} I\left(W_{2} ; \mathbf{Z}_{1 i} \mid U_{i}\right)+\sum_{i=1}^{\Delta_{2}}\left(H\left(\mathbf{Z}_{2 i}\right)-H\left(\mathbf{Z}_{2 i} \mid \mathbf{X}_{2 i}\right)\right)+\Delta \epsilon_{2} \\
= & \sum_{i=1}^{\Delta_{1}} I\left(W_{2} ; \mathbf{Z}_{1 i} \mid U_{i}\right)+\sum_{i=1}^{\Delta_{2}} I\left(\mathbf{Z}_{2 i} ; \mathbf{X}_{2 i}\right)+\Delta \epsilon_{2} \\
\leq & \sum_{i=1}^{\Delta_{1}} I\left(W_{2} \mathbf{X}_{1 i} ; \mathbf{Z}_{1 i} \mid U_{i}\right)+\sum_{i=1}^{\Delta_{2}} I\left(\mathbf{Z}_{2 i} ; \mathbf{X}_{2 i}\right)+\Delta \epsilon_{2} \\
= & \sum_{i=1}^{\Delta_{1}} I\left(\mathbf{X}_{1 i} ; \mathbf{Z}_{1 i} \mid U_{i}\right)+\sum_{i=1}^{\Delta_{2}} I\left(\mathbf{Z}_{2 i} ; \mathbf{X}_{2 i}\right)+\Delta \epsilon_{2}
\end{aligned}
$$

The final equality follows since $W_{2} \bullet\left(\mathbf{X}_{1 i}, U_{i}\right) \bullet \mathbf{Z}_{1 i}$. The necessary condition ( $7-8)$ now follows from the convexity of the region defined in $(7-8)$. The sufficiency of the condition follows from arguments based on superposition coding [2]. The proof is omitted.

We now derive the necessary and sufficient conditions for the case $X \bullet Y \ominus Z$. In this case, the weaker receiver has a later decoding deadline as compared to the stronger receiver.

Theorem 6. A necessary and sufficient condition for feasible scheduling is if $\left(\frac{w_{1}}{\Delta}, \frac{w_{2}}{\Delta}\right)$ lies in the closure of regions defined by

$$
\begin{aligned}
\frac{w_{1}}{\Delta} & <\frac{\Delta_{1}}{\Delta} I^{(1)}(X ; Y \mid U) \\
\frac{w_{2}}{\Delta} & <\frac{\Delta_{1}}{\Delta} I^{(1)}(U ; Z)+\frac{\Delta_{2}}{\Delta} I^{(2)}(X ; Z)
\end{aligned}
$$

where the mutual informations $I^{(1)}, I^{(2)}$ are evaluated with respect to the distributions $p_{U, X}^{(1)}(\cdot, \cdot)$ and $p_{X}^{(2)}(\cdot)$ respectively. By Caratheodeory's theorem ([2]), it suffices to choose $\left|\mathcal{U}_{i}\right|=$ $\min (|\mathcal{X}|,|\mathcal{Y}|,|\mathcal{Z}|)$.

Proof: The entropy of message $W_{1}$ can be bounded as

$$
\begin{aligned}
H & \left(W_{1}\right)=H\left(W_{1} \mid W_{2}\right) \leq I\left(W_{1} ; \mathbf{Y}_{1} \mid W_{2}\right)+\Delta_{1} \epsilon_{1} \\
& =H\left(\mathbf{Y}_{1} \mid W_{2}\right)-H\left(\mathbf{Y}_{1} \mid W_{1}, W_{2}\right)+\Delta_{1} \epsilon_{1} \\
& =H\left(\mathbf{Y}_{1} \mid W_{2}\right)-H\left(\mathbf{Y}_{1} \mid W_{1}, W_{2}, \mathbf{X}_{1}\right)+\Delta_{1} \epsilon_{1} \\
& =H\left(\mathbf{Y}_{1} \mid W_{2}\right)-H\left(\mathbf{Y}_{1} \mid W_{2}, \mathbf{X}_{1}\right)+\Delta_{1} \epsilon_{1} \\
& =\sum_{i=1}^{\Delta_{1}} H\left(\mathbf{Y}_{1 i} \mid W_{2}, \mathbf{Y}_{1}^{i-1}\right)-H\left(\mathbf{Y}_{1 i} \mid W_{2}, \mathbf{X}_{1}, \mathbf{Y}_{1}^{i-1}\right)+\Delta_{1} \epsilon_{1} \\
& \stackrel{(a)}{=} \sum_{i=1}^{\Delta_{1}} H\left(\mathbf{Y}_{1 i} \mid W_{2}, \mathbf{Y}_{1}^{i-1}\right)-H\left(\mathbf{Y}_{1 i} \mid W_{2}, \mathbf{X}_{1 i}, \mathbf{Y}_{1}^{i-1}\right)+\Delta_{1} \epsilon_{1} \\
& =\sum_{i=1}^{\Delta_{1}} I\left(\mathbf{X}_{1 i} ; \mathbf{Y}_{1 i} \mid W_{2}, \mathbf{Y}_{1}^{i-1}\right)+\Delta_{1} \epsilon_{1}
\end{aligned}
$$

The equality (a) follows from the memoryless property of the channel. The entropy of message $W_{2}$ is bounded as

$H\left(W_{2}\right)=I\left(W_{2} ; \mathbf{Z}_{1}, \mathbf{Z}_{2}\right)+H\left(W_{2} \mid \mathbf{Z}_{1}, \mathbf{Z}_{2}\right)$ 


$$
\begin{aligned}
& \leq I\left(W_{2} ; \mathbf{Z}_{1}, \mathbf{Z}_{2}\right)+\Delta \epsilon_{2} \\
& =I\left(W_{2} ; \mathbf{Z}_{1}\right)+I\left(W_{2} ; \mathbf{Z}_{2} \mid \mathbf{Z}_{1}\right)+\Delta \epsilon_{2} \\
& =\sum_{i=1}^{\Delta_{1}} I\left(W_{2} ; \mathbf{Z}_{1 i} \mid \mathbf{Z}_{1}^{i-1}\right)+H\left(\mathbf{Z}_{2} \mid \mathbf{Z}_{1}\right)-H\left(\mathbf{Z}_{2} \mid \mathbf{Z}_{1}, W_{2}\right)+\Delta \epsilon_{2}{ }^{\mathrm{W}} \\
& \leq \sum_{i=1}^{\Delta_{1}} I\left(W_{2}, \mathbf{Z}_{1}^{i-1} ; \mathbf{Z}_{1 i}\right)+H\left(\mathbf{Z}_{2}\right)-H\left(\mathbf{Z}_{2} \mid \mathbf{Z}_{1}, W_{2}, \mathbf{X}_{2}\right)+\Delta \epsilon_{2} \\
& \stackrel{(b)}{=} \sum_{i=1}^{\Delta_{1}} I\left(W_{2}, \mathbf{Z}_{1}^{i-1} ; \mathbf{Z}_{1 i}\right)+H\left(\mathbf{Z}_{2}\right)-H\left(\mathbf{Z}_{2} \mid \mathbf{X}_{2}\right)+\Delta \epsilon_{2} \\
& =\sum_{i=1}^{\Delta_{1}} I\left(W_{2}, \mathbf{Z}_{1}^{i-1} ; \mathbf{Z}_{1 i}\right)+\sum_{i=1}^{\Delta_{2}} H\left(\mathbf{Z}_{2 i} \mid \mathbf{Z}_{2}^{i-1}\right) \\
& -\sum_{i=1}^{\Delta_{2}} H\left(\mathbf{Z}_{2 i} \mid \mathbf{Z}_{2}^{i-1}, \mathbf{X}_{2}\right)+\Delta \epsilon_{2} \\
& \leq \sum_{i=1}^{\Delta_{1}} I\left(W_{2}, \mathbf{Z}_{1}^{i-1} ; \mathbf{Z}_{1 i}\right)+\sum_{i=1}^{\Delta_{2}} H\left(\mathbf{Z}_{2 i}\right)-\sum_{i=1}^{\Delta_{2}} H\left(\mathbf{Z}_{2 i} \mid \mathbf{X}_{2 i}\right) \\
& +\epsilon_{2}^{\Delta_{2}} \\
& =\sum_{i=1}^{\Delta_{1}} I\left(W_{2}, \mathbf{Z}_{1}^{i-1} ; \mathbf{Z}_{1 i}\right)+\sum_{i=1}^{(c)} I\left(\mathbf{X}_{2 i} ; \mathbf{Z}_{2 i}\right)+\Delta \epsilon_{2} \\
& \leq \sum_{i=1}^{\Delta_{1}} I\left(W_{2}, \mathbf{Y}_{1}^{i-1} ; \mathbf{Z}_{1 i}\right)+\sum_{i=1}^{\Delta_{2}} I\left(\mathbf{X}_{2 i} ; \mathbf{Z}_{2 i}\right)+\Delta \epsilon_{2}
\end{aligned}
$$

The equality (b) follows from the memoryless property of the channel. The inequality (c) follows from the data processing inequality since $\left(W_{2}, \mathbf{Z}_{1}^{i-1}\right) \odot\left(W_{2}, \mathbf{Y}_{1}^{i-1}\right) \odot \mathbf{Z}_{1 i}$. The necessary condition $(9-10)$ now follows from the convexity of the region defined in $(9-10)$. The sufficiency of the condition follows from arguments based on superposition coding [2]. The proof is omitted.

\section{APPENDIX: MAX-FLOW FOR POLYMATROIDAL FLOW NETWORKS}

Let $\mathcal{G}=(\mathcal{V}, \mathcal{E})$ represent a directed graph with the set $\mathcal{V}$ of vertices and the set $\mathcal{E}$ of edges . Let $s$ represent a source vertex and $\mathcal{T}$ represent a set of sink vertices. When $\mathcal{T}$ contains a single vertex, we call that vertex $t$. We recall max-flow min-cut results for a graph with polymatroidal flow constraints from [8].

\section{A. Polymatroid Flow Networks}

A polymatroid $(\mathcal{S}, \rho)$ is defined by a finite set of elements $\mathcal{S}$ and a function $\rho: 2^{\mathcal{S}} \rightarrow \mathbb{R}^{+}$satisfying the properties

$$
\begin{aligned}
\rho(\phi) & =0 \\
\rho(X) & \leq \rho(Y) \quad(X \subseteq Y \subseteq \mathcal{S}) \\
\rho(X \cup Y)+\rho(X \cap Y) & \leq \rho(X)+\rho(Y)
\end{aligned}
$$

where $X, Y \subseteq \mathcal{S}$. We are concerned with polymatroids whose elements are the edges of a graph and define a polymatroid flow network as follows. For each node $u \in \mathcal{V}$, is specified a capacity function $\beta_{u}$. The function $\beta_{u}$ satisfies the properties (11-13) with respect to the set of incoming edges $I_{u}$ of the node $u$. Thus $\left(I_{u}, \beta_{u}\right)$ is a polymatroid. A flow in the network is an assignment of real numbers to the edges of the network. We let the flow be represented by a function $f: \mathcal{E} \rightarrow \mathbb{R}^{+}$ ${ }_{2}$ which extends to sets of $\mathcal{E}$ in a natural way, i.e.,

$$
\begin{aligned}
f(\phi) & =0, \\
f(X) & =\sum_{e \in X} f(e) \quad(\phi \neq X \subseteq \mathcal{E})
\end{aligned}
$$

Such an extended flow function is said to be feasible if

$$
\begin{aligned}
f\left(I_{u}\right) & =f\left(O_{u}\right) \quad u \neq s, u \notin \mathcal{T} \\
f(X) & \leq \beta_{u}(X) \quad \forall u, X \subseteq I_{u} \\
f(e) & \geq 0 \quad \forall e \in \mathcal{E}
\end{aligned}
$$

The equation (14) imposes flow conservation at each node other than the source and the sink nodes, (15) enforces that capacity constraints are satisfied on the sets of edges and (16) requires that the flow through each edge be non-negative. For a vertex $u \in \mathcal{V}$, let $f(u)$ denote the net outgoing flow from $u$, i.e., $f(u)=f\left(O_{u}\right)-f\left(I_{u}\right)$.

Suppose we partition the vertex set $\mathcal{V}$ into two sets $\mathcal{W}$ and $\mathcal{W}^{c}$. The value the cut with respect to this partition is defined as

$$
C\left(\mathcal{W}, \mathcal{W}^{c}\right) \triangleq \sum_{u \in \mathcal{W}^{c}} \beta_{u}\left(I_{u} \cap \mathcal{E}\left(\mathcal{W}, \mathcal{W}^{c}\right)\right)
$$

Define

$$
\min -\operatorname{cut}(s, t)=\min _{\mathcal{W}}\left\{C\left(\mathcal{W}, \mathcal{W}^{c}\right): s \in \mathcal{W}, t \in \mathcal{W}^{c}\right\}
$$

It is clear that the net flow $f(s)$ from $s$ to $t$ is upper bounded by $\min$-cut $(s, t)$. It was shown in [8] that min-cut $(s, t)$ is also achievable. We recall Theorem 7.1 from [8] with slight changes in notation.

Theorem 7 (Max-flow min-cut [8]). There exists a flow $f$ from $s$ to $t$ such that

$$
f(s)=\min -\operatorname{cut}(s, t)
$$

\section{REFERENCES}

[1] C. Martel, " Preemptive Scheduling with Release Times, Deadlines and Due Times", Journal of the ACM, vol. 29, issue 3, pp.812-829, Jul 1982.

[2] T Cover, J Thomas, "Elements of Information Theory", Second Edition, Wiley Publishers.

[3] E. Uysal-Biyikoglu and A. El Gamal, " On Adaptive Transmission for Energy-efficiency in Wireless Data Networks", IEEE Transactions on Information Theory, vol. 50, issue 12, pp.3081-3094, Dec 2004.

[4] R. Berry and R. Gallager, "Buffer control for communication over fading channels ", Proc. ISIT 2000, Sorrento, Italy, June 2000, pp. 409.

[5] A. Fu, E. Modiano, and J. Tsitsiklis, "Optimal energy allocation and admission control for communications satellites", Proc. IEEE INFOCOM, New York, June 2002, vol. 2, pp. 648650.

[6] M. J. Neely, "Energy Optimal Control for Time Varying Wireless Networks", IEEE Transactions on Information Theory, vol. 52, no. 7, pp. 2915-2934, July 2006.

[7] A. Tarello, J. Sun, M. Zafer, E. Modiano, "Minimum Energy Transmission Scheduling subject to Deadline Constraints", Proc. WiOpt 2005, Trentino, Italy, April 2005.

[8] E. L. Lawler and C. U. Martel, "Computing Maximal "Polymatroidal" Network Flows", Mathematics of Operations Research, vol. 7, no. 3, Aug. 1982 